EESTI NSV TEADUSTE AKADEEMIA TOIMETISED. 25. KOIDE FOOSIKA * MATEMAATIKA. 1976, NR. 3

ИЗВЕСТИЯ АКАДЕМИИ НАУК ЭСТОНСКОИ ССР. ТОМ 25 ФИЗИКА * МАТЕМАТИКА. 1976, № 3

\title{
ВЛИЯНИЕ ПРИМЕСНЫХ НОСИТЕЛЕЙ НА СЕГНЕТОЭЛЕКТРИЧЕСКИЕ СВОЙСТВА УЗКОЩЕЛЬНЫХ ПОЛУПРОВОДНИКОВ
}

В схеме вибронной теории рассмотрено влияние дополнительных носителей примесного происхождения на сегнетоэлектрические свойства узкощельных полупроводников. Выполнены иллюстрирующие расчеты на ЭВМ. Примесные носители могут вызвать существенный сдвиг температуры Кюри и изменить форму температурных зависимостей низкосимметричного искажения решетки и частоты мягкой моды в сегнстофазе.

На основании вибронной теории сегнетоэлектрических фазовых переходов типа смещения (см., напр., $\left[{ }^{1,2}\right]$ ) получает естественное объяснение зависимость сегнетоэлектрических характеристик кристалла от концентрации носителей в нем. Для широкощельных диэлектрических систем в $\left.{ }^{3}\right]$ было показано, что при малой концентрации примесей их действие на точку Кюри $\left(T_{c}\right)$ сводимо главным образом к эффективному ослаблению межзонного электрон-фононного взаимодействия носителями примесного происхождения. Аналогичный механизм, в связи с возникновением неравновесного числа носителей, приводит к понижению точки Кюри под действием поглощаемого света $\left[{ }^{4}\right]$. Все же абсолютные величины подобного типа эффектов остаются в широкощельных системах небольшими.

Существенно более заметного влияния примесных носителей на сегнетоэлектрические свойства кристалла следует ожидать для узкощельных полупроводников. Изучению этого вопроса и посвящена настоящая работа (см. также $\left.\left[{ }^{5}\right]\right)$. В данном случае температурные зависимости входят в теорию непосредственно через изменения заселенности двух активных электронных зон (см. по этому поводу [ $\left.{ }^{1}\right]$ ), и вклад примесных носителей может быть большим.

Вибронная теория еще не сравнивалась всесторонне с экспериментом для конкретного полупроводника. Однако в $\left[{ }^{6}\right]$ найдено, что зависимость частоты мягкой моды в парафазе от температуры, согласно этой теории, описывает удовлетворительно экспериментальные данные по SnTe. B $\left[{ }^{7,8}\right]$ статическая диэлектрическая постоянная и частота мягкой моды определены для смешанной системы $n-\mathrm{Pb}_{1-x} \mathrm{Sn}_{x} \mathrm{Te}$ из экспериментов по магнитоплазменному отражению. Оказалось, что вибронная теория передает хорошо наблюдаемые зависимости от $x$ (через величину затравочной щели $\Delta$ ) и концентрации носителей. Эти работы, а также [ $\left.{ }^{9}\right]$, выявили, в частности, существенную зависимость $T_{c}$ сегнетоэлектрика-полупроводника от концентрации носителей в экспериментальном плане. Резкая зависимость $T_{c}$ кристалла $\mathrm{SnTe}$ от концентрации носителей была объяснена на основании вибронной теории в [ $\left.{ }^{10}\right]$. 
Будем считать, что концентрация примесей (доноров или акцепторов) в сегнетоэлектрике-полупроводнике с узкой запретной щелью не слишком велика. Тогда затравочную частоту $(\omega)$ активной колебательной ветви $(\vec{q} \rightarrow 0)$ и константу межзонного вибронного взаимодействия $(V)$ можно принять неизменными. Для двух электронных зон, из которых нижняя при $T=0$ заполнена $N$ электронами *, вводится эффективная щель $\Delta$ (см. $\left.\left[{ }^{11,12}\right]\right)$ и считается, что $N_{0}$ примесей образуют локальный уровень, отстоящий от потолка валентной зоны на б. Случаи акцепторов и доноров симметричны. Аналогично $\left[{ }^{1,11}\right]$ свободная энергия для кристалла с примесями может быть теперь записана в виде

$$
\begin{aligned}
F(T, y)= & \mu \sum_{\sigma=1,2,3} n_{\sigma}-k_{B} T N \sum_{\sigma=1,2} \ln \left(1+\mathrm{e}^{\frac{\mu-\bar{\varepsilon}_{\sigma}(y)}{k_{B} T}}\right)- \\
& -k_{B} T N_{0} \ln \left(1+\mathrm{e}^{\frac{\mu-\delta}{k_{B} T}}\right)+\frac{M \omega^{2}}{2} y^{2} .
\end{aligned}
$$

Здесь $y$ - нормальная координата активной предельной моды колебаний, индексы $\sigma=1,2$ относятся к двум зонам с перенормированными электрон-фононным взаимодействием энергиями

$$
\bar{\varepsilon}_{\sigma}=\frac{\Delta}{2} \mp \frac{1}{2} \sqrt{\Delta^{2}+\frac{4 V^{2}}{N} y^{2}} ;
$$

$n_{\sigma}(\sigma=3$ отвечает примесному уровню) - соответствующие числа электронов. Химический потенциал системы $\mu$ определяется условиями

$$
\sum_{\sigma} n_{\sigma}= \begin{cases}N & \text { для акцепторов, } \\ N+N_{0} & \text { для доноров, }\end{cases}
$$

причем $\underset{\sigma}{\Sigma} n_{\sigma}$ выражается с помощью условия $\frac{\partial F}{\partial \mu}=0$.

Как и в беспримесном случае $[1,2,11]$, равновесное низкосимметричное искажение решетки $y_{0}$, минимизирующее $F$, определяется системой уравнений

$$
\begin{aligned}
& \frac{y_{0}^{2}(T)}{N}=\frac{V^{2}}{\left(M \omega^{2}\right)^{2}}\left[f_{1}\left(y_{0}\right)-f_{2}\left(y_{0}\right)\right]^{2}-\frac{\Delta^{2}}{4 V^{2}}, \\
& f_{\sigma}\left(y_{0}\right)=\left[1+\exp \frac{\bar{\varepsilon}_{\sigma}\left(y_{0}\right)-\mu\left(y_{0}\right)}{k_{B} T}\right]^{-1} .
\end{aligned}
$$

В парафазе $y_{0}=0$, а частота активной мягкой ветви колебаний (ее можно найти из коэффициента разложения $F(T, y)$ по $y^{2}$ около точки $y=0$ ) определяется формулой **

где

$$
\Omega_{\mathrm{Bc} .}^{2}(T)=\omega^{2}\left[1+\tau\left(f_{2}(0)-f_{1}(0)\right)\right],
$$

$$
\tau=\frac{2 V^{2}}{M \omega^{2} \Delta}
$$

* $N$-порядка числа элементарных ячеек кристалла. Для простоты электронные зоны и активное колебание считаются невырожденными.

** Формула для частоты мягкой моды в низкосимметричной фазе в связи с еe сложностью здесь не приводится. 
Точка Кюри $T_{c}$ определяется обращением в нуль частоты мягкой моды либо низкосимметричного искажения решетки. В чистом кристалле $\mu=\frac{\Delta}{2}$ и $\quad$ условие $\Omega_{\text {вс. }}^{2}\left(T_{\mathrm{c}}\right)=0$ дает $k_{B} T_{c}=\frac{\Delta}{4}[\operatorname{Arcth} \tau]^{-1}$. Введение же примесей приводит к изменению химического потенциала системы. Согласно формулам (6) и (5), это вызовет сдвиг температуры фазового перехода, изменит ход температурных зависимостей $y_{0}(T)$ и $\Omega_{\text {вс. }}(T)$ и т. д. Поскольку дальше задача аналитическому решению не поддается, она

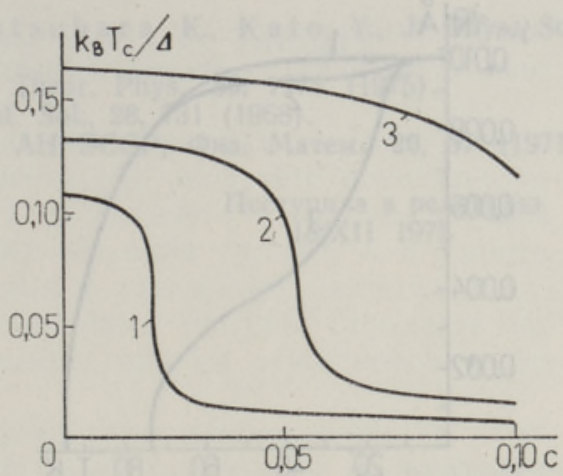

Рис. 1. Зависимость температуры Кюри от конщентрации акцепторов $(\Delta=0,1$; $\delta=0,005$ эв) при различных значениях $\tau: 1,02-(I) ; 1,05-(2)$; $1,1-(3)$. была изучена с помощью ЭВМ. Система уравнений (3)-(6) решалась численно при заданных значениях $\tau, \Delta, \delta, c=\frac{N_{0}}{N}$. Некоторые из полученных типичных результатов рассматриваются ниже.

Рис. 1 иллюстрирует зависимость температуры Кюри от концентрации акцепторов при различных значениях $\tau$. $T_{c}$ уменьшается с концентрацией $c$, поскольку приток электронов в зону проводимости и отток их из валентной зоны действуют в сторону стабилизации высокосимметричной фазы. Особенно резкие изменения в $T_{c}$ при относительно малых изменениях $c$ возможны для значений $\tau$, незначительно превышающих 1 . В $\left[{ }^{10}\right]$ найдено, что резкий спад на кривой зависимости $T_{c}$ от $c$ (типа показанного на рис. 1 для $\tau=1,02$ ) происходит при относительно больших $\Delta$, акцепторных уровнях, близких к валентной зоне, и $\tau$, близком к 1 , при концентрации, определяемой уравнением $1-\tau^{-1}-c=0$. При больших значениях $\tau$ зависимость $T_{c}$ от $c$ существенно сглаживается.

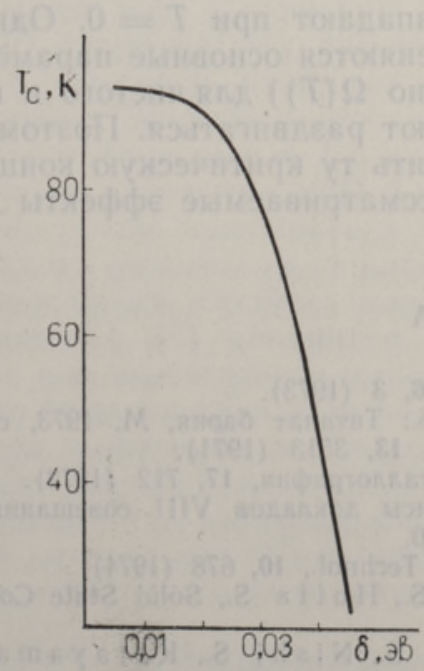

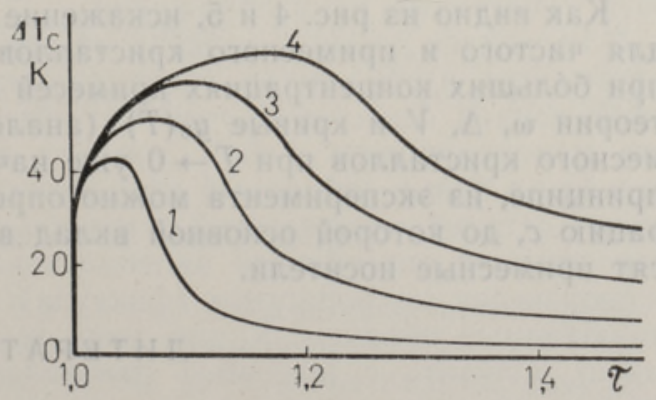

Рис. 3. Зависимость сдвига точки Кюри от характеристического параметра теории $\tau$ $(\Delta=0,05 ; \delta=0,04$ эв) при различных концентрациях доноров: $0.1-(1) ; 0,2-(2)$;

$$
0,3-(3) ; 0,4-(4) \text {. }
$$

Рис. 2. Зависимость температуры Кюри от расстояния примесного уровня до потолка валентной зоны при концентрации доноров $c=0,2 ; \Delta=0,05 \quad$ әв, $\tau=1,1$. 


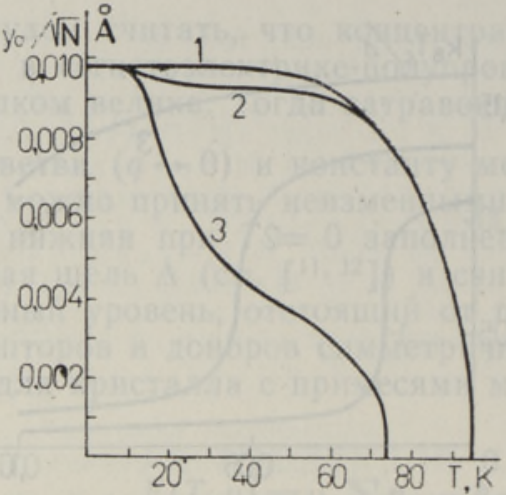

Рис. 4. Зависимость низкосимметричного искажения решетки от температуры $(\tau=1,1, \quad \Delta=0,05$, $\delta=0,045$ эв). Кривые 1 - беспримесный кристалл; $2-c=0,01$; $3-c=0,1$.

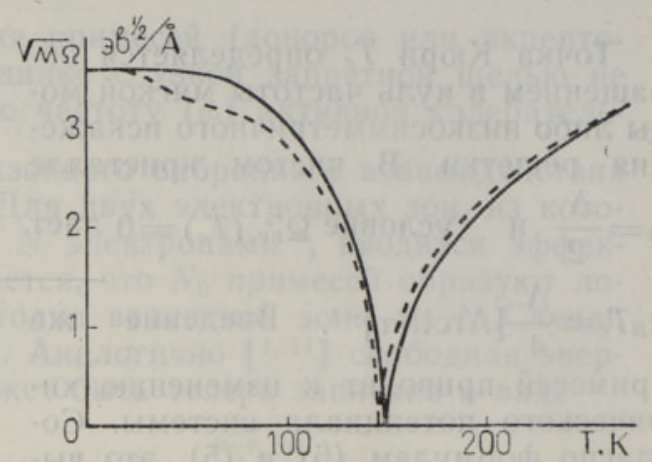

Рнс. 5. Зависимость частоты мягкой моды от температуры в пара- и сегнетофазе при $\tau=1,33, \Delta=0,05, \quad \delta=0,04$ эв. Сплошная кривая - беспримесный кристалл, пунктирная $-c=0,1$.

Сдвиг $T_{c}$ по сравнению с идеальным кристаллом сильно зависит от $\delta$ - чем ближе донорные уровни к зоне проводимости или чем ближе акцепторные уровни к валентной зоне, тем бо́льшее понижение $T_{c}$ они вызывают. Рис. 2 показывает зависимость $T_{c}$ от $\delta$. Величина сдвига $T_{c}$ зависит также сильно от $\tau$, что иллюстрируется кривыми рис. 3 . При определенном значении $\tau$, которое тем меньше, чем меньше концентрация примесей, сдвиг $T_{c}$ максимален.

Довольно значительно наличие примесей может сказаться на форме температурных зависимостей низкосимметричного искажения решетки и на частоте мягкой моды, что показывают соответственно рис. 4 и 5 . При достаточно больших концентрациях примесей искажение решетки спадает по мере приближения к $T_{c}$ медленнее. При этом низкотемпературный участок, на котором $y_{0}$ практически постоянно, сильно сужается. Аналогичные изменения появляются и в зависимости частоты мягкой моды от температуры в низкосимметричной фазе.

Как видно из рис. 4 и 5 , искажение решетки и частоты мягкой моды для чистого и примесного кристаллов совпадают при $T=0$. Однако при бо́льших концентрациях примесей изменяются основные параметры теории $\omega, \Delta, V$ и кривые $y_{0}(T)$ (аналогично $\Omega(T)$ ) для чистого и примесного кристаллов при $T \rightarrow 0$ уже начинают раздвигаться. Поэтому, в принципе, из эксперимента можно определить ту критическую концентрацию $c$, до которой основной вклад в рассматриваемые эффекты вносят примесные носители.

\section{ЛИТЕРАТУРА}

1. Krist of fel N., Kons in P., Ferroelectrics, 6, 3 (1973).

2. Кристофель Н. Н., Консин П. И., В сб.: Титанат бария, М, 1973, с. 11.

3. Кристофель Н. Н., Консин П. И., ФТТ, 13, 3513 (1971).

4. Консин П. И., Кристофель Н. Н., Кристаллография, 17, 712 (1972).

5. Консин П. И., Кристофель Н. Н., Тезисы докладов VIII совещания по теории полупроводников, Киев, 1975 , с. 100.

6. B enyon A., Grassie A. P. C., J. Vac. Sci. Technol., 10, 678 (1974).

7. Kawamura H., Katayama S., Takano S., Hotta S., Solid State Comm., $14,259(1974)$.

8. Kaw amura H., Murase K., Nishikawa $\mathrm{S}$., $\mathrm{N}$ ishi $\mathrm{S}$., Katayama $\mathrm{S}$., Solid State Comm., 17, 341 (1975). 
9. I izumi M., Hamaguchi Y., Komatsubara K., Kato Y., J. Phys. Soc. Japan, 38, 443 (1975)

10. Watarai S., Matsub ara T., Progr. Theor. Phys., 53, 1274 (1975).

11. Kristoffel N., Konsin P., Phys. Stat. Sol., 28, 731 (1968).

12. Консин П., Кристо фель Н., Изв. АН ЭССР, Физ. Матем., 20, 37 (1971).

Тартуский государственный университет

Поступила в редакцию 18/XII 1975

Ннститут физики

Академии наук Эстонской ССР

M. ITSKOVITS, P. KONSIN, N. KRISTOFFEL

\section{LISANDLAENGUKANDJATE MÕJU KITSA PILUGA POOLJUHTIDE SENJETTELEKTRILISTELE OMADUSTELE}

Vibroonteooria raames on uuritud lisanditelt pärinevate laengukandjate mõju kitsa piluga pooljuhtide senjettelektrilistele omadustele. On tehtud näitlikud arvutused elektronarvutil. Lisandlaengukandjad võivad pōhjustada olulisi Curie temperatuuri nihkeid ning muuta madalasümmeetrilise võre moonutuse ja pehme võnkumise sageduse (senjettfaasis) temperatuurisõltuvuse kuju.

\section{ITSKOVICH, P. KONSIN, N. KRISTOFFEL}

\section{INFLUENCE OF IMPURITY CARRIERS ON FERROELECTRIC PROPERTIES OF SMALL GAP SEMICONDUCTORS}

In the framework of the vibronic theory, the influence of the carriers introduced by the impurities on the ferroelectric properties of small-gap semiconductors is investigated. Illustrative computer calculations are made. Impurity-induced carriers may cause significant Curie temperature shifts and alter the form of the dependences of the lowsymmetry lattice distortion and of the soft mode frequency (in the ferroelectric phase) on the temperature. 